\title{
Biological effects of the benzotriazole ultraviolet stabilizers UV-234 and UV-320 in early-staged zebrafish (Danio rerio)
}

\author{
Xuefang Liang a, b, Ondrej Adamovsky ${ }^{\text {b, c }}$, Christopher L. Souders II ${ }^{\text {b }}$, \\ Christopher J. Martyniuk ${ }^{\mathrm{b}, *}$ \\ a Inner Mongolia Key Laboratory of Environmental Pollution Control \& Waste Resource Reuse, School of Ecology and Environment, Inner Mongolia \\ University, Hohhot, 010021, China \\ ${ }^{\mathrm{b}}$ Department of Physiological Sciences and Center for Environmental and Human Toxicology, University of Florida Genetics Institute, Interdisciplinary \\ Program in Biomedical Sciences Neuroscience, College of Veterinary Medicine, University of Florida, Gainesville, FL, 32611, USA \\ ${ }^{\mathrm{c}}$ Research Centre for Toxic Compounds in the Environment (RECETOX), Faculty of Science, Masaryk University, Brno, Czech Republic
}

\section{A R T I C L E I N F O}

\section{Article history:}

Received 22 June 2018

Received in revised form

25 October 2018

Accepted 30 October 2018

Available online 2 November 2018

\section{Keywords:}

Benzotriazole ultraviolet stabilizers

Hatching delay

Mitochondrial bioenergetics

Oxidative stress

Immune response

\begin{abstract}
A B S T R A C T
Among the benzotriazole ultraviolet stabilizers (BUVSs), UV-234 and UV-320 are frequently detected in aquatic ecosystem. Despite the fact that these chemicals are present in low ng/L levels in surface water, they show high bio-accumulation potential and pose exposure risks to aquatic organisms. However, there are limited toxicological data available in fish. In this study, zebrafish embryos were exposed to $0.01,0.1$ and $1 \mu \mathrm{M} \mathrm{UV}-234$ or UV-320 for up to 6 days. Developmental toxicity as well as effects on mitochondrial bioenergetics, immune system responses, and locomotor activity in zebrafish were measured. After UV-234 treatment $(0.1-1 \mu \mathrm{M})$, hatching time of embryos was increased compared to controls. There was also a $\sim 20-40 \%$ reduction in non-mitochondrial respiration and oligomycindependent mitochondrial respiration in embryos treated with $1 \mu \mathrm{M}$ UV-234 for 24 and $48 \mathrm{~h}$ respectively; conversely basal respiration and non-mitochondrial respiration were increased $\sim 20-30 \%$ in embryos treated with $1 \mu \mathrm{M}$ UV-320 at $48 \mathrm{~h}$. Transcript levels of sod 1 were down-regulated with BUVSs while sod2 mRNA was highly up-regulated with both UV-234 and UV-320, suggesting an oxidative damage response. Considering that mitochondrial signaling regulates innate immune pathways, we measured the expression of immune related transcripts (tlr5a, tlr5b, mmp9, il8, tnfa, cxcl-C1c, nfkb1, and ifng). Of these, only il8 and $c x c l-C 1 c$ mRNA were decreased in response to $0.1 \mu \mathrm{M}$ UV-320. To associate early molecular events with behavior, locomotor activity was assessed. UV-234 reduced larval activity in a dark photokinesis assay by $\sim 15 \%$, however behavioral responses at environmentally-relevant concentrations of BUVSs were not consistent across experiments nor BUVSs. These data suggest that BUVSs can perturb mitochondrial bioenergetics, embryonic development, and locomotor activity of zebrafish, but these responses appear to be dose-, time- and BUVSs dependent, suggesting these chemicals may have unique modes of action.
\end{abstract}

() 2018 Elsevier Ltd. All rights reserved.

\section{Introduction}

Benzotriazole ultraviolet stabilizers (BUVSs), a group of (2hydroxyphenyl) benzotriazole derivatives, have excellent absorption capacity with a full spectrum of UV-light. They are widely used

\footnotetext{
* This paper has been recommended for acceptance by Dr. Sarah Harmon.

* Corresponding author. Center for Environmental and Human Toxicology \& Department of Physiological Sciences, College of Veterinary Medicine, University of Florida, 2187 Mowry Rd. Bldg 471, Gainesville, FL, 32611, USA.

E-mail address: cmartyn@ufl.edu (C.J. Martyniuk).
}

in personal care products (e.g. cosmetics and shampoos) as well as industrial products (e.g. plastics, coatings, adhesives and construction material) to protect against ultraviolet radiation from sunlight (Nakata et al., 2009; Ruan et al., 2012). Due to their high consumption volume, BUVSs have been widely detected in surface waters, wastewaters, sewage sludge, and biota (Kameda et al., 2011; Montesdeoca-Esponda et al., 2012; Nakata et al., 2009; Nakata et al., 2010; Nakata et al., 2012; Ruan et al., 2012; Wick et al., 2016; Zhang et al., 2011; Zhao et al., 2017). Among BUVSs, 2-(benzotriazol-2-yl)-4,6-bis(2-phenylpropan-2-yl)phenol (UV-234) (Figure $\mathrm{S} 1$ ) is one of the most frequently detected BUVSs in 
environmental samples from this group of chemicals in recent years (Kameda et al., 2011; Ruan et al., 2012; Song et al., 2014; Zhao et al., 2017). It is reported that UV-234 is present in untreated and treated wastewater at concentrations in the low ng/L range ( 1.6-37.8 ng/L) (Langford et al., 2015; Lu et al., 2017b; Song et al., 2014; Zhao et al., 2017). However, lipophilic characteristics (log Kow $=7.67$ ) can lead to the presence of relatively high levels of UV234 in sediments and sewage sludge, with concentrations upwards of 363 and $607 \mathrm{ng} / \mathrm{g}$ dry weight (wt.), respectively (Kameda et al., 2011; Song et al., 2014). Similar to UV-234, 2-(benzotriazol-2-yl)4,6-ditert-butylphenol (UV-320) (Figure S1), with a log Kow value of 7.21, also shows high accumulation potential in sediments and biota. Although the use of UV-320 has been banned in Japan since 2007 due to its toxicity, persistence and bioaccumulation (Kim et al., 2012), multi-residue surveys indicate that it is still present in aquatic systems because it is tightly bound in sedimentary deposits (Cantwell et al., 2015; Nakata et al., 2009; Zhao et al., 2017). Thus, sediment appears to be a significant sink for BUVSs and this can increase the exposure risks to developing larvae in the sediment, as well as benthic aquatic organisms.

A number of studies now demonstrate that BUVSs can accumulate in blood plasma, liver, and bile of fish (Kim et al., 2011c; Lu et al., 2017a; Lu et al., 2016b; Wick et al., 2016). It is reported that concentrations of BUVSs can reach up to $316 \mathrm{ng} / \mathrm{g}$ lipid weight in fish tissues (Kim et al., 2011c). The bioaccumulation behavior of BUVSs appears to be compound- and species-specific (Kim et al., 2011a; Kim et al., 2011c; Lu et al., 2016a; Nakata et al., 2010; Nakata et al., 2012; Wick et al., 2016). Reported bioconcentration factors (BCFs) of UV-320 in carp range from 1, 380 to 8, 180 at exposure levels of $1 \mu \mathrm{g} / \mathrm{L}$ for 14 weeks, and ranged from 2, 960 to 10 , 000 at $0.1 \mu \mathrm{g} / \mathrm{L}$ for 10 weeks (Nakata et al., 2009; Nakata et al., 2010). For different fish species from an urban creek in Canada, $\log$ BAF (bioaccumulation factors) (lipid weight, l.w.) of UV-234 was determined to be 4.01 to 6.72 (Lu et al., 2016a). Thus, bioaccumulation of BUVSs can be significant and can lead to adverse effects in aquatic organisms.

Despite this knowledge, there have been limited reports on the sub-lethal impacts of BUVSs in fish or other aquatic organisms, especially UV-234 and UV-320. Some studies demonstrate that both UV-234 and UV-320 show low acute toxicity in Daphnia $\left(\mathrm{LC}_{50}>10 \mathrm{mg} / \mathrm{L}\right.$ ) (Kim et al., 2011b). In terms of chronic toxicity, it has been reported that BUVSs lack estrogenic and androgenic activities in in vitro yeast assays (Kawamura et al., 2003; Morohoshi et al., 2005). However, some BUVSs have been shown to have anti-androgenic activity (e.g. UV-328 and UV-P) (Zhuang et al., 2017) and aryl hydrocarbon receptor (AhR) ligand activity (e.g. UV-P, UV-9, UV-326, and UV-090) in vitro (Nagayoshi et al., 2014). Additionally, biological activities of BUVSs can be altered after metabolism. For example, metabolized UV-328 showed increased anti-androgenic activity in comparison with UV-328 upon CYP3A4-mediated biotransformation (Zhuang et al., 2017). As a result, toxicities of BUVSs as well as their mode of action should be investigated in vivo to better understand their response in the organism. BUVSs have been documented to activate the AHR-pathway in zebrafish embryos based on the induction of genes such as arh1, arnt2, and cyp1a1, as well as genes involved in anti-oxidant responses such as glutathione-S-transferase (gst) (Fent et al., 2014). In our previous study, zebrafish embryos exposed to 4 BUVSs (UV-234, UV-326, UV-329 and UV-P) showed altered expression of transcripts in the thyroid hormone pathway, which is important for metabolic homeostasis and development (Liang et al., 2017a). Although BUVSs have received increased attention because of their bioaccumulation and potential toxicity, knowledge as to their toxicological effects in aquatic organisms remains limited.
Aquatic pollutants that disturb the balance between production and depletion of reactive oxygen species (ROS) can induce oxidative stress. A recent study has shown that UV-234 and UV-328 can increase ROS production as well as oxidative stress related genes that included glutathione peroxidase (gpx), superoxide dismutase (sod), and catalase (cat) in freshwater green algae Chlamydomonas reinhardtii (Giraudo et al., 2017). In biological systems, mitochondrial oxidative phosphorylation is a major cellular source of ROS (Lushchak, 2011). Recent studies have shown that mitochondria participate in a broad range of innate immune pathways, functioning as signaling platforms and contributing to effector responses (West et al., 2011). Mounting evidence suggests that mitochondria facilitate antibacterial immunity by generating reactive oxygen species (ROS) and contribute to innate immune activation following cellular damage and stress (Cloonan and Choi, 2013; Lartigue and Faustin, 2013; West et al., 2011). The innate immune system has a key role in the vertebrate immune response, and is exquisitely sensitive to environmental contaminants (Bols et al., 2001). Immunotoxicity can influence the development, growth, and survival of fish (Wester et al., 1994), which can ultimately affect the stability of fish populations. In zebrafish, the active innate immune system is detectable at day 1 of embryogenesis (Trede et al., 2004). However, a functionally mature adaptive immune system is not active until 4-6 weeks post-fertilization (Trede et al., 2004). This temporal separation makes zebrafish (Danio rerio) embryos an appropriate model for studying innate immunity in vertebrates (Novoa and Figueras, 2012).

To evaluate the sub-lethal effects of BUVSs in early-staged zebrafish, we firstly assessed the developmental toxicity of UV234 and UV-320. Bioenergetic perturbations induced by exogenous chemicals during embryogenesis are expected to affect the developmental process, thus indicators to assess mitochondrial function including oxidative respiration (i.e. mitochondrial bioenergetics) and sod1 and sod2 expression levels were evaluated. Furthermore, several transcripts related to the innate immune system were measured to better address the interaction between mitochondria and the immune system. To provide relevance to higher level endpoints, we also investigated the effects of UV-234 and UV-320 on locomotor behaviors in zebrafish larvae, as early evidence of toxicity could lead to detrimental ecologically relevant responses (i.e. adverse outcome pathway).

\section{Materials and methods}

\subsection{Chemicals}

2-(benzotriazol-2-yl)-4,6-bis(2-phenylpropan-2-yl)phenol (UV234, CAS no. 70321-86-7, batch number: MKBW2452V) was purchased from Sigma-Aldrich Co. LLC (USA). 2-(benzotriazol-2-yl)4,6-ditert-butylphenol (UV-320, CAS no. 3846-71-7, purity >98\%) was purchased from Ark Pharm, Inc. (USA). Stock solutions of $0.1,1$ and $10 \mathrm{mM}$ UV-234 or UV-320 were prepared by dilution in dimethyl sulfoxide (DMSO, CAS no. 67-68-5, purity $>99.9 \%$, Sigma). The exposure solutions were prepared by adding $10 \mu \mathrm{L}$ of each stock solution into $100 \mathrm{~mL}$ of embryo rearing medium (ERM; $13.69 \mathrm{mM}$ $\mathrm{NaCl}, 0.54 \mathrm{mM} \mathrm{KCl}, 0.025 \mathrm{mM} \mathrm{Na}_{2} \mathrm{HPO}_{4}, 0.044 \mathrm{mM} \mathrm{KH}_{2} \mathrm{PO}_{4}, 1.3 \mathrm{mM}$ $\mathrm{CaCl}_{2}, 1 \mathrm{mM} \mathrm{MgSO} 4,4.17 \mathrm{mM} \mathrm{NaHCO}$, $\mathrm{pH} 7.2$ ) yielding the final concentrations of $0.01,0.1$ and $1 \mu \mathrm{M} \mathrm{UV}-234$ (equal 4.48, 44.8, and $447.6 \mu \mathrm{g} / \mathrm{L}$ ) or UV-320 (equal 3.23, 32.3, and $323.4 \mu \mathrm{g} / \mathrm{L}$ ). A vehicle treatment of DMSO served as control. The ratio of vehicle to ERM was $1: 10,000(\mathrm{vol} / \mathrm{vol})$. Considering the high accumulation potential of BUVSs in sediments and biota, exposure concentrations used in this study were higher than those occurring in the environment (in the low ng/L). 


\subsection{Experimental design}

Experimental design for exposures and endpoints are similar to that reported previously (Liang et al., 2017b). Please refer to the Supporting Information for more specific details. Briefly, adult zebrafish (ZF, Wild type $A B$ ) were randomly selected from a breeding stock and placed in two tanks to generate the fertilized embryos. The ratio was three males to one female per tank. The fertilized eggs ( 300-400) were determined to be at the same stage (shield stage, $\sim 6 \mathrm{~h}$ post fertilization, hpf) and these embryos were used in subsequent experiments. Embryos were staged as outlined previously (Kimmel et al., 1995). Ten embryos were equally distributed into one glass beaker containing $10 \mathrm{~mL}$ of each exposure solution. Five replicate beakers were performed for each condition (control, $0.01,0.1$, and $1 \mu \mathrm{M} \mathrm{UV-234}$ or UV-320). Embryos were maintained in an incubator at $26.0 \pm 1{ }^{\circ} \mathrm{C}$ with a photoperiod of 14:10 h light: dark. For the duration of the experiment, a static waterborne exposure was conducted over $96 \mathrm{~h}$ with $90 \%$ water renewal with the chemical each day.

\subsection{Embryo development}

Developmental experiments were performed according to Liang et al. (2017b). Briefly, embryos were transferred to wells of a 96well culture plate and were treated with the solvent control or one of the three concentrations of UV-234 or UV-320. UV-234 and UV-320 experimental groups were measured separately on two 96well plates. Each treatment included $n=24$ embryos. The 96-well plate was incubated at $26 \pm 1{ }^{\circ} \mathrm{C}$ in the EVOS FL Cell Imaging System (Thermo Scientific, USA) and images of each well were automatically acquired every hour for $96 \mathrm{~h}$. Developmental parameters that included hatching time, mortality, and malformation were manually scored using the method of Reimers et al. (2004).

\subsection{Analysis of metabolic rates}

Extracellular flux analyzer assay was optimized according to Souders et al. (2018). Please refer to the Supporting Information for more specific details. Two separate experiments were conducted for oxygen consumption rate (OCR) for both chemicals. After exposure for either 24 or $48 \mathrm{~h}$, one embryo ( $n=5$ total) from separate biological replicates from each treatment was selected for mitochondrial respiration assessment using the $\mathrm{XF}^{\mathrm{e}} 24$ Extracellular Flux Analyzer (Agilent Seahorse XF Technology, USA). Basal respiration [defined as (final basal OCR measurement)], mitochondrial respiration [defined as (final basal OCR measurement - final $\mathrm{NaN}_{3}$ OCR measurement)], and non-mitochondrial respiration [defined as (final $\mathrm{NaN}_{3}$ OCR measurement)] were calculated as per Seahorse XF Cell Mito Stress Test Kit User Guide (User Guide Kit 103015-100, Agilent). Here, basal respiration is baseline OCR data for each individual collected over a number of pre-defined cycles. Oligomycin is then used to assess ATP dependent respiration in the embryos (oligomycin-induced ATP production). Following $\mathrm{NaN}_{3}$, oxidative phosphorylation was completely blocked and non-mitochondrial respiration (e.g. oxygenase activity) was determined. The relevance of these endpoints for mitochondrial function and bioenergetics are provided in Souders et al. (2018), but in short, constitute endpoints that suggest mitochondrial dysfunction following treatment with specific-acting mitochondrial toxicants.

\subsection{Gene expression analysis}

After $6 \mathrm{~d}$ exposure, larvae in each group were collected and 5 larval fish were pooled into one centrifuge tube from each beaker $(n=5 /$ condition). Extraction of RNA and cDNA synthesis were performed according to Liang et al. (2017b). Primer sets for target genes were collected from literature (Table S1). The genes were selected to map the modulation of several important inflammatory mediators like interferon, gamma (ifng), tumor necrosis factor a (tnfa), chemokine (C-X-C motif) ligand 8a (il8), chemokine (C-X-C motif) ligand $18 \mathrm{~b}$ ( $\mathrm{cxcl}-\mathrm{C1c}$ ), and matrix metallopeptidase 9 ( $\mathrm{mmp9}$ ) upon BUVSs exposure (Jin et al., 2008). To better understand to upstream regulatory mechanism, we included regulators of the innate immunity toll-like receptors (tlr5a and tlr5b) and nuclear factor of kappa light polypeptide gene enhancer in B-cells 1 ( $n f k b 1$ ), both trigger and regulate mentioned immune mediators and are often highly expressed in fish immune cells upon stimulation (Hayashi et al., 2001). Transcript levels of superoxide dismutase (sod1 and sod2) were also measured as an indicator of oxidative stress. Three reference genes (beta actin, $\beta$-actin; ribosomal subunit 18, rps18; and ribosomal protein $\mathrm{L} 13 \alpha, r p l 13 a$ ) were combined for normalization. Real-time PCR was performed according to Liang et al. (2017b). More specific details are provided in the Supporting Information.

\subsection{Dark photokinesis behavioral assay}

After exposure for $5 d$ in beakers, larval zebrafish were individually transferred into a 96-well culture plate and placed in a Noldus DanioVision Observation Chamber (Noldus Information Technology, Netherlands). For each treatment, 24 fish were assessed for behavior in mid-afternoon. After being acclimated at $26^{\circ} \mathrm{C}$ the instrument for $2 \mathrm{~h}$, the activities of fish were recorded by an infrared analog camera within the Danio Vision observation chamber, and the resulting video was collected and analyzed using the EthoVision software version 12.0 (Noldus Information Technology, Leesburg, VA). Larvae were tracked following a standard 50 min "white light routine" of alternating $10 \mathrm{~min}$ periods of light and dark beginning with a dark period (i.e. dark photokinesis). Analysis profiles were generated in EthoVision for distance moved and activity (i.e. percent pixel change across wells over time with an activity threshold of 16 to remove background noise) and an excel file was exported for further analysis.

\subsection{Statistical analysis}

Statistical analysis of variance was performed using SPSS (version 17.0). The normality of data was assessed using the Kolmogorov-Smirnov test and logarithmic transformation was performed prior to ANOVA to ensure data conformed to assumptions. The Levene's test of homogeneity of variance was used to test for adherence to one-way analysis of variance (ANOVA) assumptions. Hatching time was analyzed using one-way ANOVA followed by Dunnett's post-hoc test was used to compare each treatment with the control. For hatching rates and survival rates, the Log-rank (Mantel-Cox) test was used to compare the differences between treatments and the control. Mitochondrial respiration was analyzed by two-way ANOVA and comparison of means between each treatment and the control was carried out using Dunnett's test as post hoc test. All quantitative data above are expressed as mean \pm standard error (SEM). The qPCR results were analyzed by Kruskal-Wallis followed by Dunn's multiple comparison tests. For behavior analysis, exported data were first pooled into 5 sections according to each of the dark/light periods measured. Treatments included DMSO control, $0.01,0.1$ and $1 \mu \mathrm{M}$ BUVSs. Data were analyzed using one-way ANOVA followed by Holm-Sidak's multiple comparison tests. A probability of $\mathrm{p}<0.05$ was considered to be statistically significant. All figures were prepared using Graph-Pad Prism version 6.0 (GraphPad Sofware Inc., La Jolla, CA, USA). 


\section{Results}

\subsection{Developmental toxicity of UV-234 and UV-320 in zebrafish embryos}

Mortality and hatching rate were not different between treatments and the control during $96 \mathrm{~h}$ exposure to UV-234 or UV-320 (Table S2 and Figure S2). However, there was an increase in hatching time for the UV-234 exposure groups (i.e. took longer to hatch). The average hatching time of zebrafish embryos in the control group was $74.5 \mathrm{~h}$, while that in treatments was $78.95-79.68 \mathrm{~h}$, ranging from $5.97 \%$ to $6.95 \%(d f=84, F=7.7$, $p=0.0001$ ) (Fig. 1A). No differences were detected among doses, suggesting that this may be associated with a threshold effect as opposed to a dose dependent response at these concentrations. There were no differences observed for embryos exposed to UV$320(d f=84, F=1.47, p=0.23)$ (Fig. 1B).

\subsection{UV-234 and UV-320 exposure altered mitochondrial bioenergetics}

To address any effects of UV-234 and UV-320 on mitochondrial bioenergetics, oxygen consumption rate (OCR) was measured in intact zebrafish embryos after 24 and 48 -h exposure, respectively (Fig. 2A and B). After 24-h UV-234 exposure, non-mitochondrial respiration was significantly decreased following $1 \mu \mathrm{M}$ UV-234 treatment $(d f=32, F=3.52, p=0.02)$. Conversely, there were no changes in basal respiration $(d f=32, F=3.45, p=0.17)$ nor mitochondrial respiration $(d f=32, F=2.68, p=0.66)$ in any treatment compared to control (Fig. 3C-E, upper graph). For the 48-h UV-234 exposed embryos, mitochondrial respiration was significantly inhibited in $1 \mu \mathrm{M}$ UV-234 treatment $(\mathrm{df}=32 ; F=2.68, p=0.025)$. There were no differences detected in fish for basal respiration $(d f=32, F=3.45, p=0.06)$ nor non-mitochondrial respiration for any treatment compared to the control $(d f=32, F=3.52, p=0.16)$ (Fig. 2C-E, upper graph).

For the 24-h UV-320 treatments, there were no changes in basal respiration $(d f=32, F=2.02, p=0.9)$, mitochondrial respiration $(d f=32, F=1.56, p=0.69)$, nor non-mitochondrial respiration $(d f=32, F=1.91, p=0.68)$ (Fig. 2C-E, lower graph). However, for the 48-h exposure to UV-320, basal respiration ( $\mathrm{df}=32, F=2.02$, $p=0.024)$ and non-mitochondrial respiration $(\mathrm{df}=32, F=1.91$, $p=0.028$ ) were significantly increased in embryos at $1 \mu \mathrm{M} \mathrm{UV}-320$ group; no difference was detected in fish for mitochondrial respiration among groups ( $d f=32, F=1.56, p=0.059$ ) (Fig. $2 C-E$, lower graph). Taken together, there is some evidence that these compounds affect respiration and this effect appears to be dependent upon the dose and the time point examined.

\section{3. $U V-234$ and $U V-320$ exposure affected the expression of genes related to oxidative stress}

To test the hypothesis that BUVSs induce mitochondrial damage response, the mRNA levels of sod 1 and sod2 were measured in larvae following exposure to UV-234 and UV-320 (Fig. 3). After 6d exposure of UV-234, a significant decrease in sod1 mRNA levels were detected in fish from the $0.01 \mu \mathrm{M}$ treatment $(-1.87$-fold, $p=0.045$ ) (Fig. 3A). Conversely, levels of sod2 mRNA were upregulated following $1 \mu \mathrm{M}$ UV-234 exposure (15.1-fold, $p=0.01$ ) (Fig. 3B). After 6-d exposure to UV-320 treatment, levels of sod1 were significantly decreased in fish from the $0.1 \mu \mathrm{M}$ UV-320 treatment ( -6.4 -fold, $p=0.017$ ) (Fig. 3C). Conversely, levels of sod2 were increased in fish from the $0.1 \mu \mathrm{M}$ UV-320 treatment (7.7fold, $p=0.002$ ) (Fig. 3D). Fold change on a $\log 2$ scale depicts changes in gene expression, and relative expression was compared to the control group to reveal differences among the three treatments.

\subsection{UV-320, but not UV-234, altered the expression of genes related to the immune system}

We examined transcript levels for genes involved in toll-like receptor (TLR) signaling pathways that included toll-like receptors (tlr5a and $t l r 5 b$ ), immune response regulator $n f k b 1$, proinflammation cytokines (ifng, tnfa), chemokines (il8, cxcl-C1c) and matrix metalloprotease mmp9 (Fig. 4 and Figure S3). All transcripts tested were not altered in abundance following UV-234 exposure (Figure S3). In the UV-320 exposure experiments, tlr5a, tlr5b, nfkb1, ifng, tnfa and mmp9 were not different in relative abundance in the zebrafish larvae (Fig. 4A-E and H). However, the level of il8 was decreased in fish exposed to $0.1 \mu \mathrm{M}$ UV-320 group compared to the control group (7.9-fold, $p=0.02$ ) (Fig. 4F). Similarly, levels of chemokine $\mathrm{cxcl}-\mathrm{C} 1 \mathrm{c}$ were significantly decreased in fish exposed to $0.1 \mu \mathrm{M}$ UV-320 group compared to the control (2.8-fold, $p=0.03$ ) (Fig. 4G).

\subsection{Locomotor behavior of zebrafish larvae was affected by both $U V-234$ and UV-320}

Behavioral responses were subtle for both UV stabilizers and were dependent upon the dose and chemical. In the first $10 \mathrm{~min}$, decreased activity of larval zebrafish pre-adapted to darkness was detected in larvae exposed to UV-234 ( $d f=36, F=34, p<0.0001$ ) (Figure S4A, B). In the following light-dark cycle, as expected with
(A)

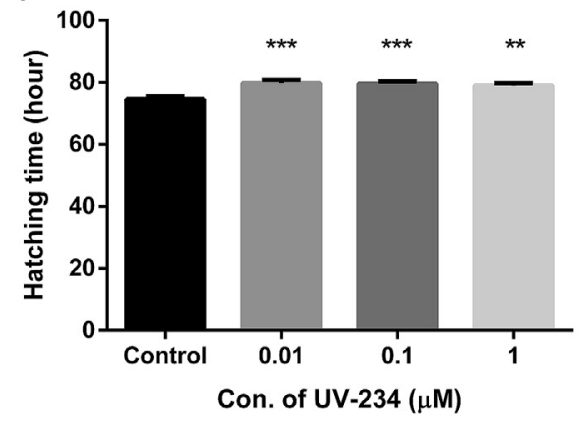

(B)

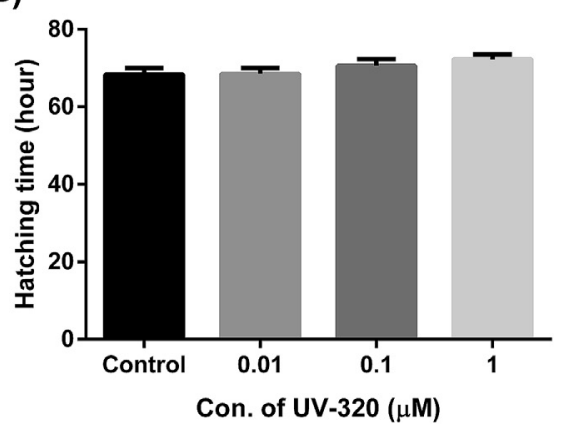

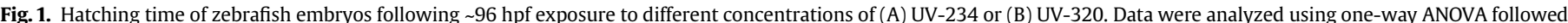

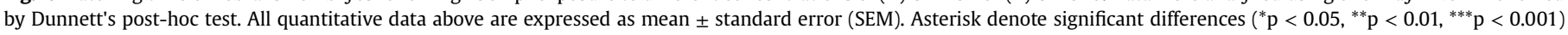
between treatments and controls. 
(A)
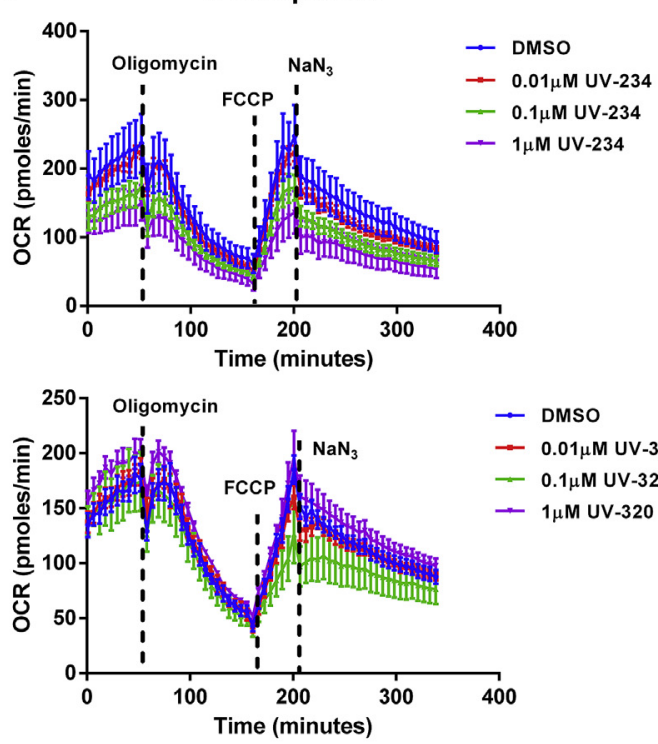

(C)
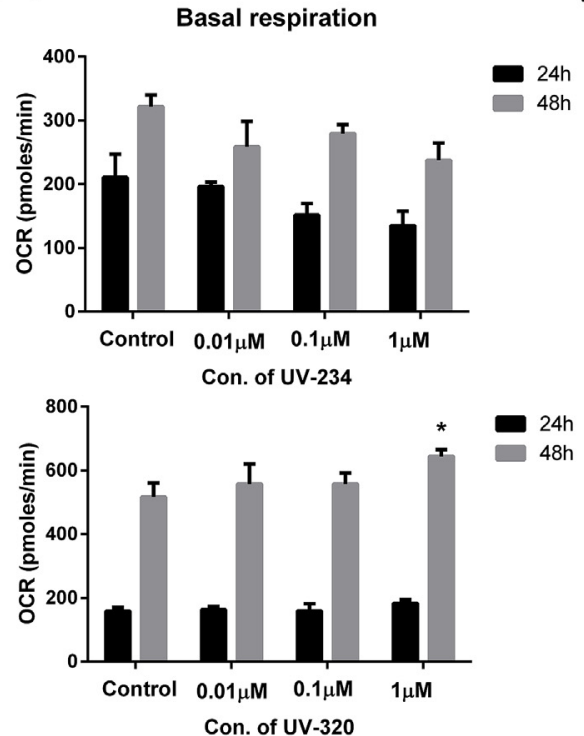

(B) 48h exposure
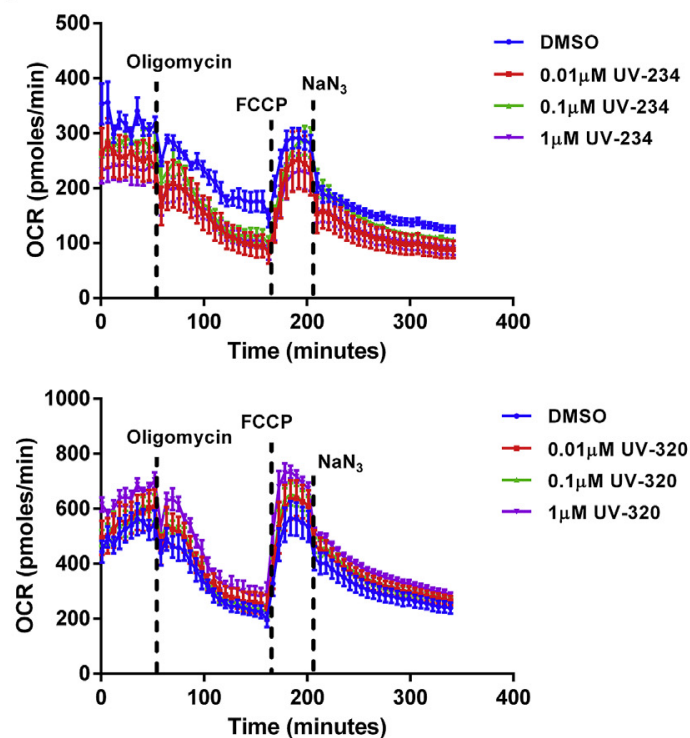

(D)

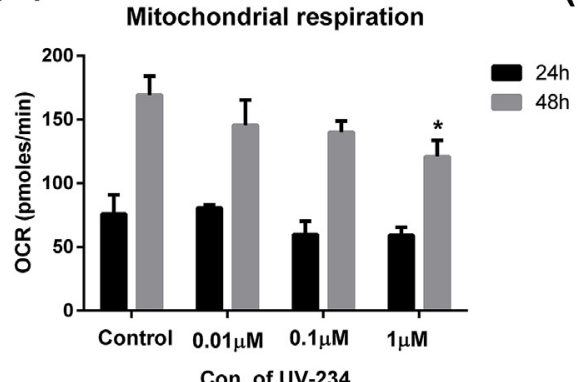

(E)
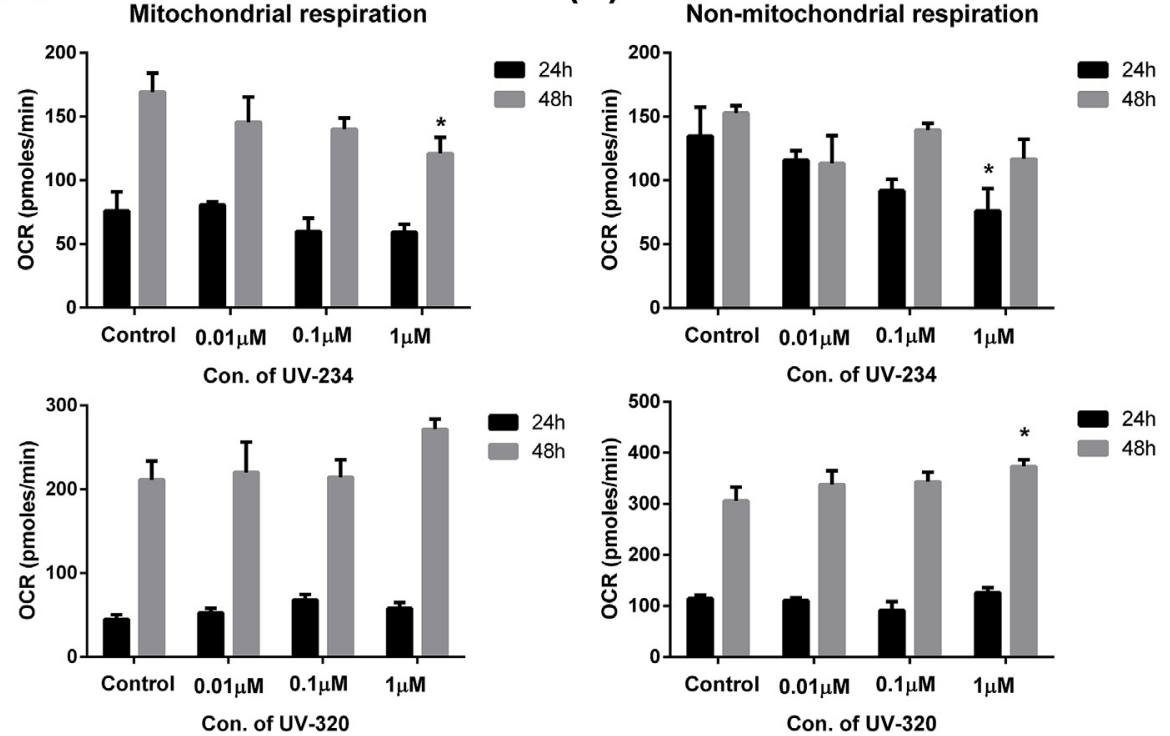

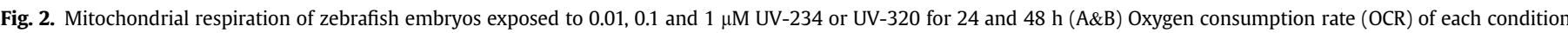

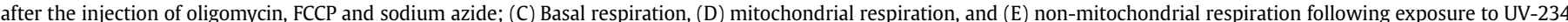

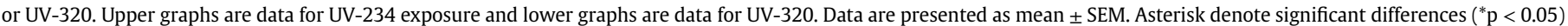
between treatments and controls. Detailed description of each endpoint measured can be found in Souders et al. (2018).

dark photokinesis, locomotor activity increased with loss of illumination, but there was no difference in the activity of larvae in UV234 groups compared to the control (Figure S4A, B). In contrast, a decrease in movement (distance moved) was detected in larvae following treatment to $1 \mu \mathrm{M} \mathrm{UV}-234(d f=36, F=4.1, p=0.028)$ in 20-30 min following light extinction (Fig. 5A and B). During subsequent light (30-40 min), the total distance moved in larvae was different than controls ( $d f=36, F=33, p=0.003)$. Distance moved was increased in response to $0.01 \mu \mathrm{M}$ while distance moved was lower in larvae exposed to $0.1 \mu \mathrm{M}$ UV-234 compared to control (Fig. 5A and B). Taken together, there was some evidence that UV234 altered behavior; however there was no discernible pattern in response.

For UV-320 treatments, increased activity of larval zebrafish pre-adapted to darkness was detected in larvae exposed to $1 \mu \mathrm{M}$ ( $d f=36, F=37, p<0.0001$ ) compared to the control (assessed in first $10 \mathrm{~min}$ ) (Figure S4C, D). Increased distance moved for larvae was also observed with $1 \mu \mathrm{M}$ UV-320 exposure $(d f=36, F=12$, $p<0.0001$ ) in the first $10 \mathrm{~min}$ (dark) (Fig. 5C and D). In response to illumination, larvae increased their distance moved in the $0.1 \mu \mathrm{M}$ UV-320 treatment ( $d f=36, F=6.3, p=0.004$ ) compared to control (10-20 min) (Fig. 5C and D). However, following a light-dark cycle, fish exhibited a decrease in distance moved in the $1 \mu \mathrm{M}$ UV-320 treatment $(d f=36, F=4.3, \quad p=0.016)$ in $30-40 \mathrm{~min}$ (light) (Fig. 5C and D). Taken together, changes in activity was small and dependent upon the dose.

\section{Discussion}

BUVSs are emerging pollutants of concern which have been widely detected in aquatic environments (Nakata et al., 2009; Song et al., 2014). Depending on their structure, BUVSs and their 
(A)

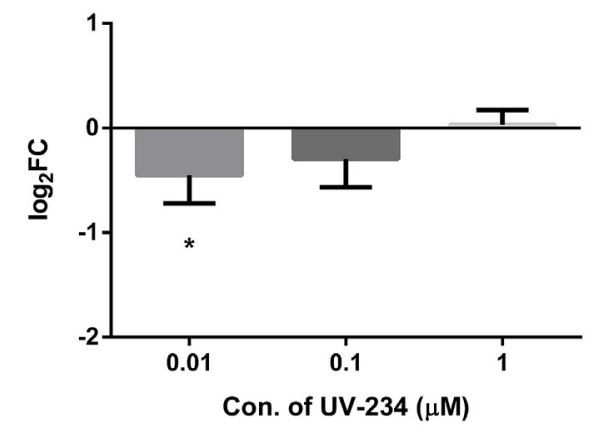

(C)

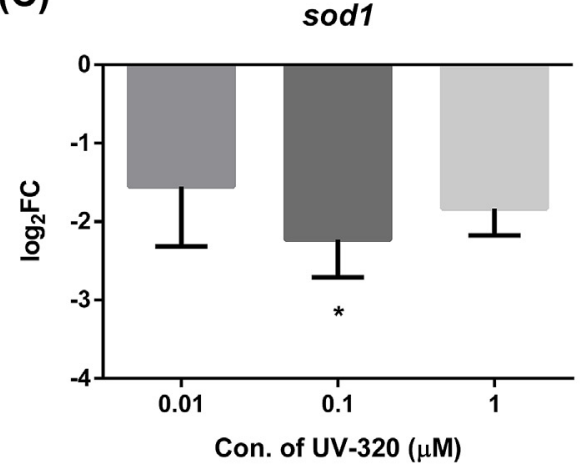

(B)

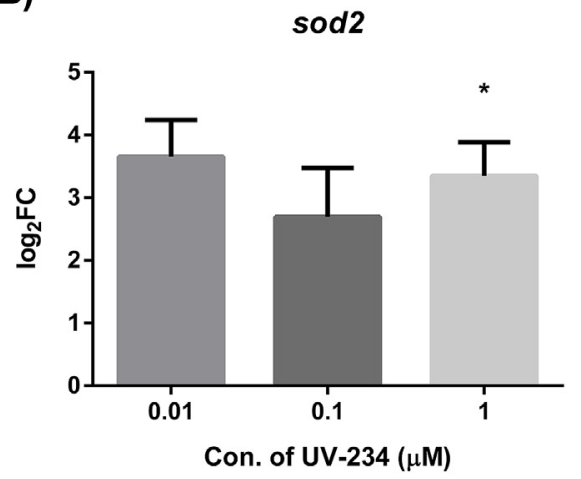

(D)

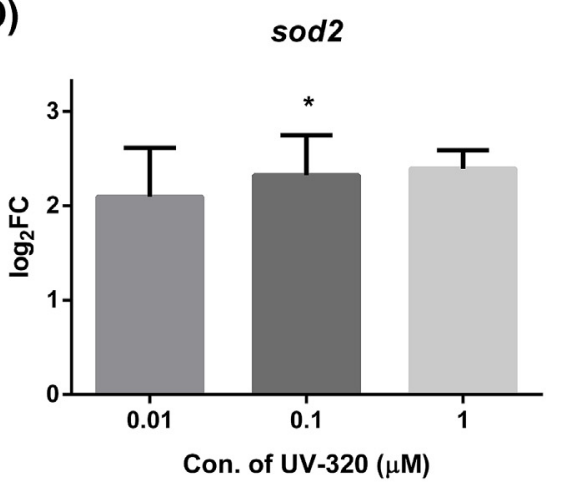

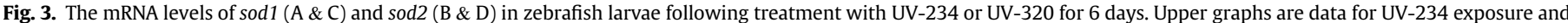

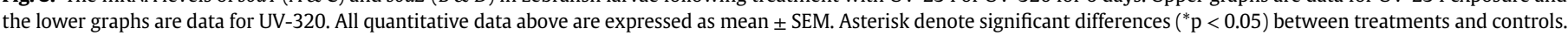

(A)

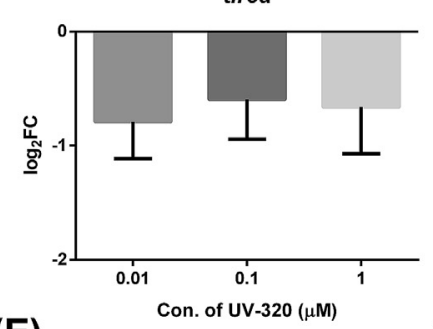

(E)

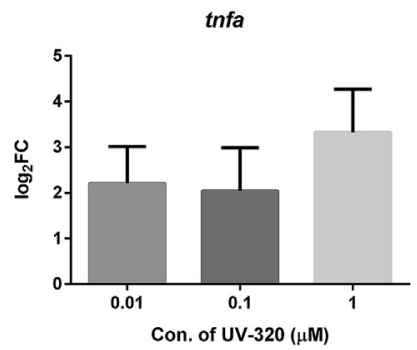

(B)

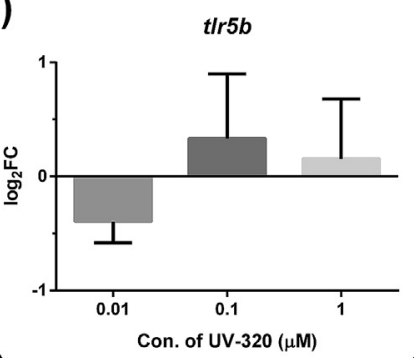

(F)

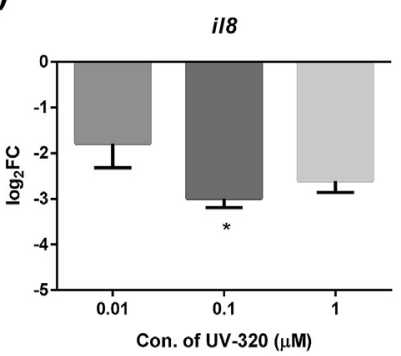

(C)

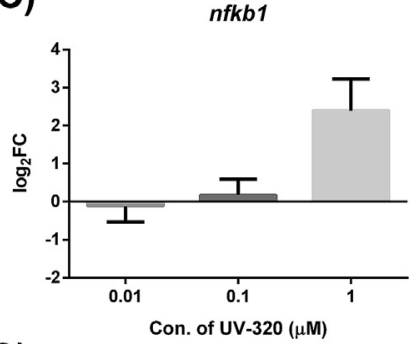

(G)

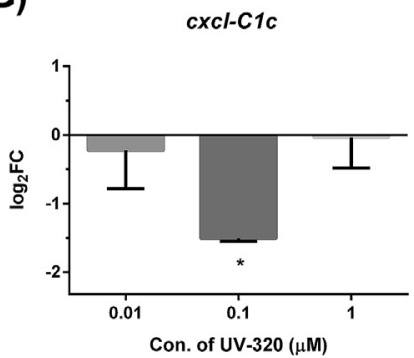

(D)

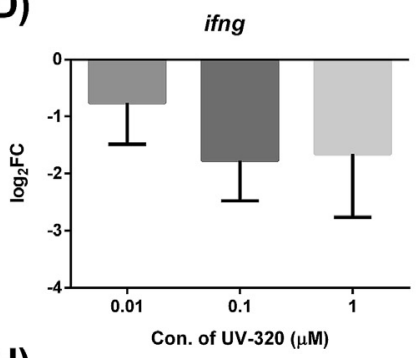

(H)

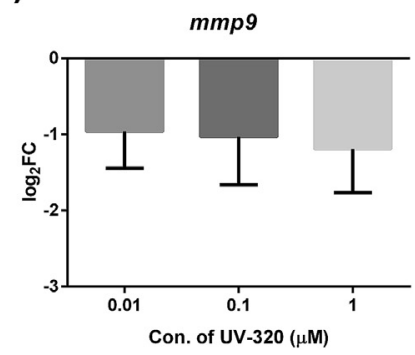

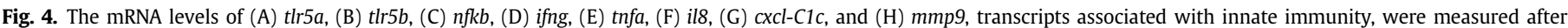

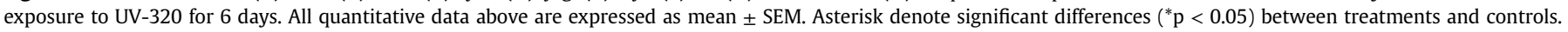

metabolites can exert structure-dependent effects in both mammals (Nagayoshi et al., 2014) and aquatic organisms (Fent et al., 2014; Liang et al., 2017a; Zhuang et al., 2017). Despite several reports on their endocrine disrupting effects in mammals and fish (Fent et al., 2014; Nagayoshi et al., 2014; Zhuang et al., 2017), studies investigating their potential for developmental toxicity and immunotoxicity have not yet been conducted. In this study, zebrafish were exposed to two commonly detected BUVSs, UV-234 and UV-320, for up to 6 days and developmental toxicity, mitochondrial bioenergetics, behavior, and molecular endpoints related to the oxidative stress and the immune system were measured. 
(A)

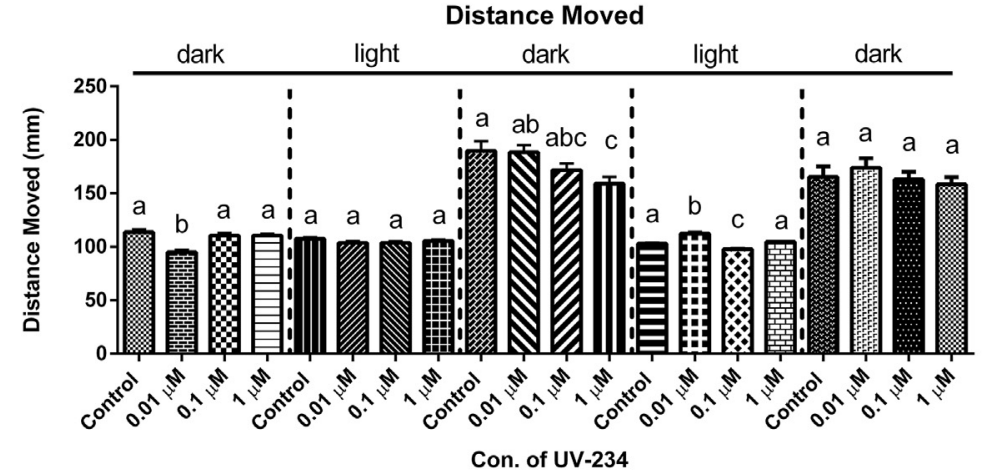

(C)

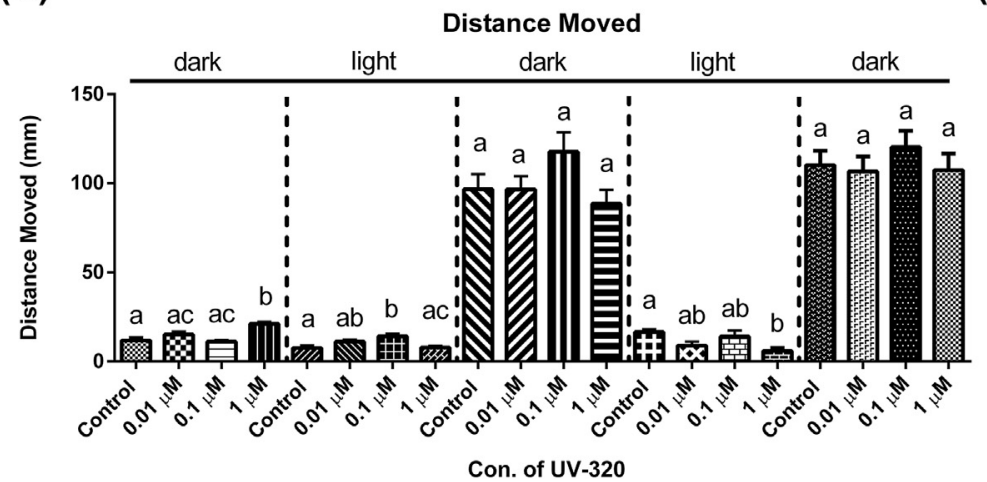

(B)

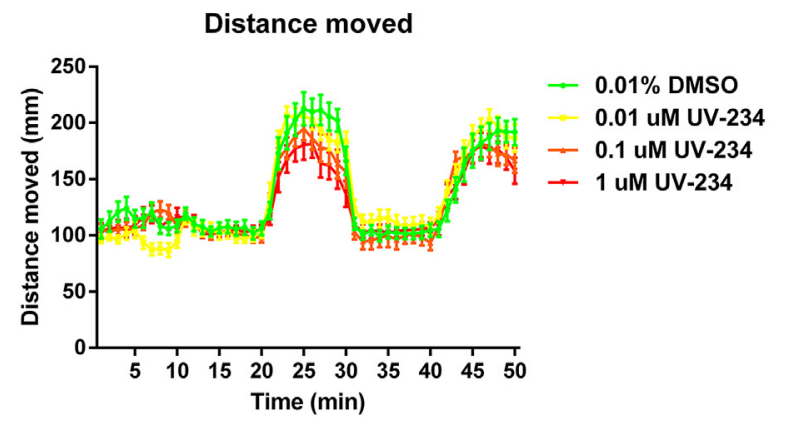

(D)

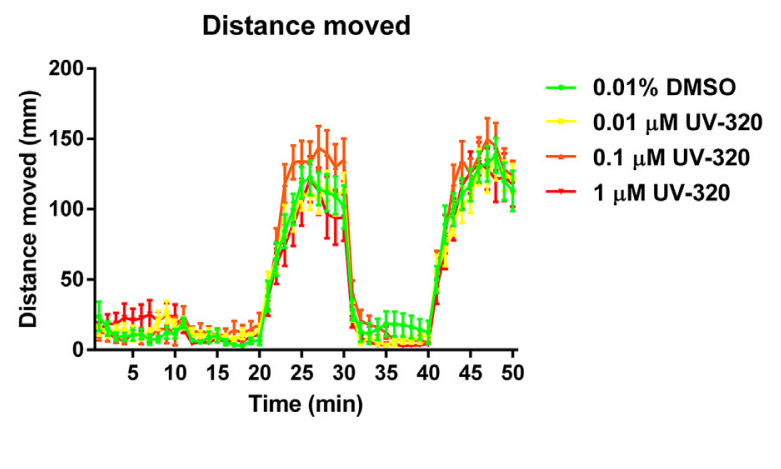

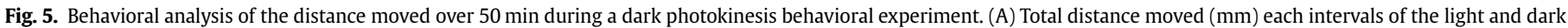

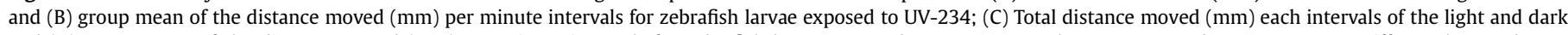

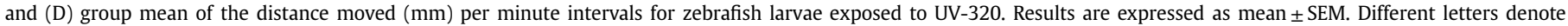
significant differences among groups within an interval $(P<0.05)$.

\subsection{Hatching time of zebrafish embryos is affected differently by BUVSS}

In this study, hatching time was significantly increased (i.e. delayed hatching) with exposure to UV-234, but this was not the case for UV-320 treatments. As pointed out in our previous study, time to hatch may be a sensitive indicator for adverse effects in fish embryos exposed to chemicals (Liang et al., 2017b). The mechanism for the delayed hatching may involve a change in the elasticity of the chorion or in chorionic hatching enzyme function (Winnicki et al., 1970), although this must be rigorously tested with the chemical under study. Another hypothesis related to the delay in hatching may involve oxidative respiration in developing fish; exposure to hypoxic environments delayed hatching of sheepshead minnow (Cyprinodon variegatus) embryos (Hendon et al., 2008). In the present study, oxygen consumption rate (OCR) was decreased by UV-234 while there was an increase in OCR with UV-320. Previous studies have suggested that chemical-induced alterations in mitochondrial bioenergetics in zebrafish embryos may contribute to developmental toxicity (e.g. curved tails or abnormal hatching time) (Wang et al., 2018; Zhang et al., 2017). Noteworthy is that hatching delay is reported to be associated with the interference in the thyroid hormone (TH) system (Timme-Laragy et al., 2006). In our previous study, UV-234 was shown to alter the expression of the thyroid hormone pathway in zebrafish embryos (Liang et al., 2017a). Thus, we hypothesize that mitochondrial dysfunction and disruptions in TH system induced by UV-234 may both contribute to the hatching delay. However, further studies are required to elucidate the exact role of each in a biologically complex process.

\subsection{BUVSs induce oxidative damage in developing zebrafish at the transcript level}

Mitochondrial dysfunction can lead to increased reactive oxygen species (ROS) and apoptosis. Mitochondrial oxidative phosphorylation is a major cellular source of ROS and levels of ROS are not only determined by production, but also by the rate of ROS degradation or inactivation (Mailloux and Harper, 2012). ROS can be converted into hydrogen peroxide $\left(\mathrm{H}_{2} \mathrm{O}_{2}\right)$ in the mitochondrial matrix by superoxide dismutase 2 (SOD2) $\mathrm{H}_{2} \mathrm{O}_{2}$ can then freely cross mitochondrial membranes or can be further detoxified by additional mitochondrial antioxidant enzymes, such as glutathione peroxidase (GPX). In the cytosol, superoxide is converted by SOD1 into $\mathrm{H}_{2} \mathrm{O}_{2}$, which is further detoxified by the peroxisomal enzyme catalase (Li et al., 2013). Redox imbalance can be therefore generated when a cell accumulates an excessive amount of ROS which overwhelms antioxidant defenses.

In this study, oxidative and non-mitochondrial respiration of zebrafish embryos were suppressed by $1 \mu \mathrm{M}$ UV-234 exposure. Noteworthy was that mRNA level of sod2 was increased at $1 \mu \mathrm{M} U \mathrm{UV}-$ 234 in larval fish ( $6 \mathrm{dpf}$ ). The upregulation of sod2 suggests that the antioxidant defense system was activated due to redox imbalance (Knecht et al., 2013) and it is hypothesized that this could be due to the impaired bioenergetics observed with BUVSs. In mammal and fish cells, $10 \%$ of cellular oxygen uptake is due to non-mitochondrial (e.g. oxidase), rather than mitochondrial use of oxygen for respiration (Rolfe et al., 1999). The increase in non-mitochondrial oxygen consumption has been considered to be a protective mechanism by some to minimize oxidative stress (Raftery et al., 2017). 
Similar to the pattern observed with UV-234, the mRNA levels of sod1 were down-regulated while that of sod2 were upregulated in fish exposed to $0.1 \mu \mathrm{M}$ UV-320. This may be associated with the upregulation of basal and non-mitochondrial respiration at $1 \mu \mathrm{M}$ UV-320, indicative of redox imbalance. The elevated sod 2 and decreased sod $1 \mathrm{mRNA}$ levels suggest that there is a molecular response to increased mitochondrial ROS production as expected. Previous studies have shown that there can be increased levels of sod2 mRNA in response to oxidative stress caused by environmental stress or chemicals in zebrafish (Cai et al., 2012; Tiedke et al., 2013). Moreover, Zou et al. (2017) reported that isoniazide induces oxidative stress in developing zebrafish by increasing ROS, and that this was associated with a decrease in sod1 mRNA levels. Our data indicate that UV-320 may affect mitochondrial bioenergetics and induce oxidative stress in developing zebrafish.

\subsection{The effects of BUVSs on locomotor activity in zebrafish}

Chemicals that impair mitochondrial function may also underlie abnormal development in fish embryos (Bestman et al., 2015). In early life stages of fish, behavioral responses can act as sensitive indicators of toxicant exposure (Liang et al., 2017b). Here, activity of zebrafish larvae was assessed using a standard light-dark challenge. The activity of zebrafish larvae is characterized by higher activity in the dark compared to light at early stages of development. This hyperactive state has been termed the visual motor response (VMR) (Mueller and Neuhauss, 2012). Hypoactivity following UV-234 exposure in the larvae was observed in these experiments and implies that the chemical caused a decrease in visual sensitivity. To be noted, during the second light-dark cycle $(40-50 \mathrm{~min})$, the behavior of UV-234 exposed larvae became indistinguishable from that of control animals. Thus, at these environmentally relevant concentrations, it is somewhat unclear how BUVSs affect behavior and the response may be clutch/dose specific. In a previous study of zebrafish larvae exposed to cocaine, loss of a difference in locomotor activity was also observed after longer exposure regimes; in that study, the possible reason was proposed to be the elimination of accumulated cocaine in the brain of fish (Kirla et al., 2016). The effect of UV-234 was subtle, and the loss of a response at the end of the assay may be attributed to the biotransformation or habituation in the assay. Nevertheless, these results suggest that UV-234 may influence neuronal pathways that mediate behavioral responses to light in zebrafish larvae.

In contrast to UV-234, UV-320 did not significantly alter locomotor activity of zebrafish larvae in the dark cycle, but it altered larvae travel distance in light periods. Moreover, in continuous darkness (first 10 min of the assay), increased activity of larvae was observed in the highest dose. Naturally, zebrafish prefer dark areas over light areas. Larval fish also display a startle response when exposed to a sudden change in light intensity (Colwill and Creton, 2011). It has been reported that stress responsive behavior is highly sensitive to neuroactive chemicals (e.g. diazepam and carbamazepine) (Brandão et al., 2013). However, to date, few studies have demonstrated that BUVSs induce neurotoxicity in fish at the physiological or molecular level. Liang et al. (2016) demonstrated that $>0.42 \mu \mathrm{M}$ benzotriazole can induce alterations of brain proteomic profiles related to neurotoxicity in female Chinese rare minnows (Gobiocypris rarus). However, currently strong evidence is lacking for physiological or behavioral responses to support neuroactivity of benzotriazole and its derivatives (e.g. BUVSs). Additional studies investigating other behaviors such as anxiety or feeding behavior may reveal evidence for neurological or energyrelated limitations.

\subsection{The immune system: a potential target for BUVSs toxicity?}

Apart from producing ATP, controlling calcium homeostasis, and regulating apoptosis, mitochondria also act as a sensor for stressors and can initiate inflammatory signals in the cell by activating the innate immune system (Lartigue and Faustin, 2013; West et al., 2011). Studies show that dysfunction in mitochondria is related to inflammatory diseases and disorders (reviewed in Cloonan and Choi, 2013). Altered oxygen consumption in the embryos as well as changes in the mRNA levels of sod1 and sod2 in response to BUVSs in the present study led us to the hypothesis that the innate immune system may also be affected by BUVSs toxicity.

To explore the potential for immunotoxicity of BUVSs, we measured transcripts of several key inflammatory markers (tnfa and ifng) and signals that attract other immune cell types to the site of inflammation - chemokines il-8, cxcl-C1c and mmp9. All of these genes are triggered by toll like receptor (TLR) dependent NF- $\mathrm{B}$ signaling pathway, that function as enhancers and regulators of genes involved in innate immune response (Hayashi et al., 2001). Despite the similarity in the structure of UV-234 and UV-320, only UV-320 showed any evidence for an immunomodulatory response. All concentration of UV-234 tested did not interfere with the expression of transcripts investigated, but fish in the $0.1 \mu \mathrm{M} \mathrm{UV}-320$ group showed evidence for downregulation of chemokines ( $\mathrm{cxcl}$ C1c and il8). Both of these transcripts are regulated by nuclear factor NF- $\kappa$ B. However, we did not observe any changes in the transcriptomic level of $n f k b 1$ in our exposures. In contract to chemokines, transcriptomic levels of pro-inflammatory cytokines ( $t n f a$ and ifng) and upstream regulators ( $n f k b 1$ and $t \operatorname{lr} 5 a / b)$ were not changed, indicating that the signaling pathway TLR- NF- $\mathrm{B}$ -proinflammatory cytokines may not be modified by UV-320.

Both downregulated cytokines Cxcl-c1c (also known as Cxcl18b) and IL-8( $\mathrm{Cxcl} 8)$ are chemokines molecules, that were shown to be a reliable inflammatory markers. These CXC subgroup of chemokines are the key controllers of neutrophil chemotaxis in mammals and in teleost fish including zebrafish (de Oliveira et al., 2013; Deng et al., 2013). They are produced by innate immunity cell (e.g. macrophages), but also by non-infected cells that immediate surround the infected/inflamed tissue (Torraca et al., 2017). They control several biological function including homestasis, cell recruitment and immune function (Russo et al., 2014). Dysregulation of these chemokines have the direct effect on the ability of the host to respond the microbial infection and to eliminate the pathogens (Torraca et al., 2014). In addition, Cxcl signaling axis significantly contribute to disease-associated processes, including tissue injury, fibrosis, angiogenesis and tumorigenesis (Russo et al., 2014; Torraca et al., 2014). Inhibition of cxcl-C1c and il8 by UV-320 suggest that chemokines may be potential molecular targets for BUVSs.

\subsection{Conclusions}

In conclusion, both UV-234 and UV-320 can affect mitochondrial bioenergetics and the expression of transcripts related to oxidative stress (i.e. sod1 and sod2) in zebrafish embryos. However, our data support the notion that these UV stabilizers may also act differently in terms of their mechanisms. We hypothesize that UV234 can affect mitochondrial bioenergetics and cause hatching delay in zebrafish embryos. However, UV-320 is proposed to alter immune response via transcriptional mechanisms following disturbing mitochondrial bioenergetics in zebrafish. The underlying mechanisms may contribute to the differential activity behaviors of larval zebrafish in response to changes in illumination. Further research in neuro-toxicological effects of BUVSs after longer-term exposure is needed to provide additional evidence for the behavioral response observed here. Additional molecular targets should 
also be investigated as potential biomarkers for the exposure to BUVSs in fish.

A final point to make is that we understand little regarding the toxicity and biological effects of biotransformation products of BUVSs. Zhuang et al. (2017) observed that UV-328 had an enhanced anti-androgenic effect following CYP3A4-mediated biotransformation, and showed that hydroxylation is the dominant process involved in the biotransformation. Recently, Damalas et al. (2018) assessed the toxicity of 3 benzotriazoles that included 1-H-benzotriazole (BT), 4-Methyl-1-H-benzotriazole (4-MeBT), and 5methyl-1-H-benzotriazole (5-MeBT) as well as their biotransformation products in zebrafish (Danio rerio) larvae; it was demonstrated that parent compounds had a higher toxicity potential compared with their biotransformation products, and 4-MeBT showed the highest toxicity potential. However, no data on transformation products of UV-234 and UV-320 exist to date and this remains a significant knowledge gap moving forward in assessing environmental risks associated with BUVSs.

\section{Acknowledgements}

The authors have no conflict of interest to declare. We thank Edward Flynn and the Animal Care Services at University of Florida for animal husbandry. This work was supported by the National Natural Science Foundation of China $(21507064,21866024)$, Marie Skłodowska-Curie actions no.707241- European Commission Research Program, the research infrastructure projects from Czech Ministry of Education (LM2015051 and CZ.02.1.01/0.0/0.0/16_013/ 0001761), and the University of Florida, College of Veterinary Medicine. The support provided by China Scholarship Council during a visit of Xuefang Liang (No. 201608155003) to the University of Florida is acknowledged.

\section{Appendix A. Supplementary data}

Supplementary data to this article can be found online at https://doi.org/10.1016/j.envpol.2018.10.130.

\section{References}

Bestman, J.E., Stackley, K.D., Rahn, J.J., Williamson, T.J., Chan, S.S.L., 2015. The cellular and molecular progression of mitochondrial dysfunction induced by 2,4dinitrophenol in developing zebrafish embryos. Differentiation 89, 51-69.

Bols, N.C., Brubacher, J.L., Ganassin, R.C., Lee, L.E.J., 2001. Ecotoxicology and innate immunity in fish. Dev. Comp. Immunol. 25, 853-873.

Brandão, F.P., Rodrigues, S., Castro, B.B., Gonçalves, F., Antunes, S.C., Nunes, B., 2013. Short-term effects of neuroactive pharmaceutical drugs on a fish species: biochemical and behavioural effects. Aquat. Toxicol. 144-145, 218-229.

Cai, G., Zhu, J., Shen, C., Cui, Y., Du, J., Chen, X., 2012. The effects of cobalt on the development, oxidative stress, and apoptosis in zebrafish embryos. Biol. Trace Elem. Res. 150, 200-207.

Cantwell, M.G., Sullivan, J.C., Katz, D.R., Burgess, R.M., Bradford Hubeny, J., King, J. 2015. Source determination of benzotriazoles in sediment cores from two urban estuaries on the Atlantic Coast of the United States. Mar. Pollut. Bull. 101, 208-218.

Cloonan, S.M., Choi, A.M.K., 2013. Mitochondria: sensors and mediators of innate immune receptor signaling. Curr. Opin. Microbiol. 16, 327-338.

Colwill, R.M., Creton, R., 2011. Imaging escape and avoidance behavior in zebrafish larvae. Rev. Neurosci. 22, 63-73.

Damalas, D.E., Bletsou, A.A., Agalou, A., Beis, D., Thomaidis, N.S., 2018. Assessment of the acute toxicity, uptake and biotransformation potential of benzotriazoles in zebrafish (Danio rerio) larvae combining HILIC-with RPLC-HRMS for highthroughput identification. Environ. Sci. Technol. 52 (10), 6023-6031.

de Oliveira, S., Reyes-Aldasoro, C.C., Candel, S., Renshaw, S.A., Mulero, V., Calado, A., 2013. Cxcl8 (IL-8) mediates neutrophil recruitment and behavior in the zebrafish inflammatory response. J. Immunol. 190, 4349-4359.

Deng, Q., Sarris, M., Bennin, D.A., Green, J.M., Herbomel, P., Huttenlocher, A., 2013. Localized bacterial infection induces systemic activation of neutrophils through Cxcr2 signaling in zebrafish. J. Leukoc. Biol. 93, 761-769.

Fent, K., Chew, G., Li, J., Gomez, E., 2014. Benzotriazole UV-stabilizers and benzotriazole: antiandrogenic activity in vitro and activation of aryl hydrocarbon receptor pathway in zebrafish eleuthero-embryos. Sci. Total Environ. 482,
$125-136$

Giraudo, M., Cottin, G., Esperanza, M., Gagnon, P., Silva, A.O., Houde, M., 2017. Transcriptional and cellular effects of benzotriazole UV stabilizers UV-234 and UV-328 in the freshwater invertebrates Chlamydomonas reinhardtii and Daphnic magna. Environ. Toxicol. Chem. 36, 3333-3342.

Hayashi, F., Smith, K.D., Ozinsky, A., Hawn, T.R., 2001. The innate immune response to bacterial flagellin is mediated by Toll-like receptor 5 . Nature 410, 1099 .

Hendon, L.A., Carlson, E.A., Manning, S., Brouwer, M., 2008. Molecular and developmental effects of exposure to pyrene in the early life-stages of Cyprinodon variegatus. Comp. Biochem. Physiol. C Toxicol. Pharmacol. 147, 205-215.

Jin, T., Xu, X., Hereld, D., 2008. Chemotaxis, chemokine receptors and human disease. Cytokine 44, 1-8.

Kameda, Y., Kimura, K., Miyazaki, M., 2011. Occurrence and profiles of organic sunblocking agents in surface waters and sediments in Japanese rivers and lakes. Environ. Pollut. 159, 1570-1576.

Kawamura, Y., Ogawa, Y., Nishimura, T., Kikuchi, Y., Nishikawa, J.-i., Nishihara, T. Tanamoto, K., 2003. Estrogenic activities of UV stabilizers used in food contact plastics and benzophenone derivatives tested by the yeast two-hybrid assay J. Health Sci. 49, 205-212.

Kim, J.W., Ramaswamy, B.R., Chang, K.H., Isobe, T., Tanabe, S., 2011a. Multiresidue analytical method for the determination of antimicrobials, preservatives, benzotriazole UV stabilizers, flame retardants and plasticizers in fish using ultra high performance liquid chromatography coupled with tandem mass spectrometry. J. Chromatogr. A 1218, 3511-3520.

Kim, J.W., Chang, K.H., Isobe, T., Tanabe, S., 2011b. Acute toxicity of benzotriazole ultraviolet stabilizers on freshwater crustacean (Daphnia pulex). J. Toxicol. Sci. 36, 247-251.

Kim, J.W., Isobe, T., Malarvannan, G., Sudaryanto, A., Chang, K.H., Prudente, M. Tanabe, S., 2012. Contamination of benzotriazole ultraviolet stabilizers in house dust from the Philippines: implications on human exposure. Sci. Total Environ. $424,174-181$

Kim, J.W., Isobe, T., Ramaswamy, B.R., Chang, K.H., Amano, A., Miller, T.M. Siringan, F.P., Tanabe, S., 2011c. Contamination and bioaccumulation of benzotriazole ultraviolet stabilizers in fish from Manila Bay, the Philippines using an ultra-fast liquid chromatography-tandem mass spectrometry. Chemosphere $85,751-758$.

Kimmel, C.B., Ballard, W.W., Kimmel, S.R., Ullmann, B., Schilling, T.F., 1995. Stages of embryonic development of the zebrafish. Dev. Dynam. 203, 253-310.

Kirla, K.T., Groh, K.J., Steuer, A.E., Poetzsch, M., Banote, R.K., Stadnicka-Michalak, J. Eggen, R.I., Schirmer, K., Kraemer, T., 2016. From the cover: zebrafish larvae are insensitive to stimulation by cocaine: importance of exposure route and toxicokinetics. Toxicol. Sci. 154, 183-193.

Knecht, A.L., Goodale, B.C., Truong, L., Simonich, M.T., Swanson, A.J., Matzke, M.M. Anderson, K.A., Waters, K.M., Tanguay, R.L., 2013. Comparative developmental toxicity of environmentally relevant oxygenated PAHs. Toxicol. Appl. Pharmacol. 271, 266-275.

Langford, K.H., Reid, M.J., Fjeld, E., Øxnevad, S., Thomas, K.V., 2015. Environmental occurrence and risk of organic UV filters and stabilizers in multiple matrices in Norway. Environ. Int. 80, 1-7.

Lartigue, L., Faustin, B., 2013. Mitochondria: metabolic regulators of innate immune responses to pathogens and cell stress. Int. J. Biochem. Cell Biol. 45, 2052-2056.

Li, X., Fang, P., Mai, J., Choi, E.T., Wang, H., Yang, X.F., 2013. Targeting mitochondrial reactive oxygen species as novel therapy for inflammatory diseases and cancers. J. Hematol. Oncol. 6, 19.

Liang, X., Li, J., Martyniuk, C.J., Wang, J., Mao, Y., Lu, H., Zha, J., 2017a. Benzotriazole ultraviolet stabilizers alter the expression of the thyroid hormone pathway in zebrafish (Danio rerio) embryos. Chemosphere 182, 22-30.

Liang, X., Souders, C.L., Zhang, J., Martyniuk, C.J., 2017b. Tributyltin induces premature hatching and reduces locomotor activity in zebrafish (Danio rerio) embryos/larvae at environmentally relevant levels. Chemosphere 189 498-506.

Lu, Z., De Silva, A.O., Peart, T.E., Cook, C.J., Tetreault, G.R., 2017a. Tissue distribution of substituted diphenylamine antioxidants and benzotriazole ultraviolet stabilizers in white sucker (Catostomus commersonii) from an urban Creek in Canada. Environ. Sci. Technol. Lett. 4, 433-438.

Lu, Z., De Silva, A.O., Peart, T.E., Cook, C.J., Tetreault, G.R., Servos, M.R., Muir, D.C., 2016a. Distribution, partitioning and bioaccumulation of substituted diphenylamine antioxidants and benzotriazole UV stabilizers in an urban creek in Canada. Environ. Sci. Technol. 50, 9089-9097.

Lu, Z., Peart, T.E., Cook, C.J., De Silva, A.O., 2016b. Simultaneous determination of substituted diphenylamine antioxidants and benzotriazole ultra violet stabilizers in blood plasma and fish homogenates by ultra high performance liquid chromatography-electrospray tandem mass spectrometry. J. Chromatogr. A 1461, 51-58.

Lu, Z., Smyth, S.A., Peart, T.E., De Silva, A.O., 2017b. Occurrence and fate of substituted diphenylamine antioxidants and benzotriazole UV stabilizers in various Canadian wastewater treatment processes. Water Res. 124, 158-166.

Lushchak, V.I., 2011. Environmentally induced oxidative stress in aquatic animals. Aquat. Toxicol. 101, 13-30.

Mailloux, R.J., Harper, M.E., 2012. Mitochondrial proticity and ROS signaling: lessons from the uncoupling proteins. Trends Endocrinol. Metabol. 23, 451-458.

Montesdeoca-Esponda, S., Sosa-Ferrera, Z., Santana-Rodriguez, J.J., 2012. On-line solid-phase extraction coupled to ultra-performance liquid chromatography with tandem mass spectrometry detection for the determination of benzotriazole UV stabilizers in coastal marine and wastewater samples. Anal. Bioanal. 
Chem. 403, 867-876.

Morohoshi, K., Yamamoto, H., Kamata, R., Shiraishi, F., Koda, T., Morita, M., 2005. Estrogenic activity of 37 components of commercial sunscreen lotions evaluated by in vitro assays. Toxicol. Vitro 19, 457-469.

Mueller, K.P., Neuhauss, S.C., 2012. Light perception: more than meets the eyes. Curr. Biol, 22, R912-R914.

Nagayoshi, H., Kakimoto, K., Takagi, S., Konishi, Y., Kajimura, K., Matsuda, T., 2014. Benzotriazole ultraviolet stabilizers show potent activities as human aryl hydrocarbon receptor ligands. Environ. Sci. Technol. 49, 578-587.

Nakata, H., Murata, S., Filatreau, J., 2009. Occurrence and concentrations of benzotriazole UV stabilizers in marine organisms and sediments from the Ariake Sea, Japan. Environ. Sci. Technol. 43, 6920-6926.

Nakata, H., Shinohara, R., Murata, S., Watanabe, M., 2010. Detection of benzotriazole UV stabilizers in the blubber of marine mammals by gas chromatography-high resolution mass spectrometry (GC-HRMS). J. Environ. Monit. 12, 2088-2092.

Nakata, H., Shinohara, R., Nakazawa, Y., Isobe, T., Sudaryanto, A., Subramanian, A. Tanabe, S., Zakaria, M.P., Zheng, G.J., Lam, P.K., Kim, E.Y., Min, B.Y., We, S.U., Viet, P.H., Tana, T.S., Prudente, M., Frank, D., Lauenstein, G., Kannan, K., 2012. Asia-Pacific mussel watch for emerging pollutants: distribution of synthetic musks and benzotriazole UV stabilizers in Asian and US coastal waters. Mar. Pollut. Bull. 64, 2211-2218.

Novoa, B., Figueras, A., 2012. Zebrafish: model for the study of inflammation and the innate immune response to infectious diseases. Adv. Exp. Med. Biol. 946 $253-275$.

Raftery, T.D., Jayasundara, N., Di Giulio, R.T., 2017. A bioenergetics assay for studying the effects of environmental stressors on mitochondrial function in vivo in zebrafish larvae. Comp. Biochem. Physiol. C Toxicol. Pharmacol. 192, 23-32.

Rolfe, D.F., Newman, J.M., Buckingham, J.A., Clark, M.G., Brand, M.D., 1999. Contribution of mitochondrial proton leak to respiration rate in working skeletal muscle and liver and to SMR. Am. J. Physiol. 276, C692-C699.

Reimers, M.J., Flockton, A.R., Tanguay, R.L., 2004. Ethanol- and acetaldehydemediated developmental toxicity in zebrafish. Neurotoxicol. Teratol. 26 769-781.

Ruan, T. Liu, R., Fu, Q., Wang, T., Wang, Y., Song, S., Wang, P., Teng, M., Jiang, G., 2012. Concentrations and composition profiles of benzotriazole UV stabilizers in municipal sewage sludge in China. Environ. Sci. Technol. 46, 2071-2079.

Russo, R.C., Garcia, C.C., Teixeira, M.M., Amaral, F.A., 2014. The CXCL8/IL-8 chemokine family and its receptors in inflammatory diseases. Expet Rev. Clin. Immunol. 10, 593-619.

Song, S., Ruan, T., Wang, T., Liu, R., Jiang, G., 2014. Occurrence and removal of benzotriazole ultraviolet stabilizers in a wastewater treatment plant in China. Environ Sci Process Impacts 16, 1076-1082.

Souders 2nd, C.L., Liang, X., Wang, X., Ector, N., Zhao, Y.H., Martyniuk, C.J., 2018 High-throughput assessment of oxidative respiration in fish embryos: advancing adverse outcome pathways for mitochondrial dysfunction. Aquat. Toxicol. 199, 162-173.
Tiedke, J., Cubuk, C., Burmester, T., 2013. Environmental acidification triggers oxidative stress and enhances globin expression in zebrafish gills. Biochem. Biophys. Res. Commun. 441, 624-629.

Timme-Laragy, A.R., Levin, E.D., Di Giulio, R.T., 2006. Developmental and behavioral effects of embryonic exposure to the polybrominated diphenylether mixture DE-71 in the killifish (Fundulus heteroclitus). Chemosphere 62, 1097-1104.

Torraca, V., Masud, S., Spaink, H.P., Meijer, A.H., 2014. Macrophage-pathogen interactions in infectious diseases: new therapeutic insights from the zebrafish host model. Dis Model Mech 7, 785-797.

Torraca, V., Otto, N.A., Tavakoli-Tameh, A., Meijer, A.H., 2017. The inflammatory chemokine Cxcl18b exerts neutrophil-specific chemotaxis via the promiscuous chemokine receptor Cxcr2 in zebrafish. Dev. Comp. Immunol. 67, 57-65.

Trede, N.S., Langenau, D.M., Traver, D., Look, A.T., Zon, L.I., 2004. The use of zebrafish to understand immunity. Immunity 20, 367-379.

Wang, X.H., Souders, C.L., Zhao, Y.H., Martyniuk, C.J., 2018. Paraquat affects mitochondrial bioenergetics, dopamine system expression, and locomotor activity in zebrafish (Danio rerio). Chemosphere 191, 106-117.

West, A.P., Shadel, G.S., Ghosh, S., 2011. Mitochondria in innate immune responses. Nat. Rev. Immunol. 11, 389-402.

Wester, P.W., Vethaak, A.D., van Muiswinkel, W.B., 1994. Fish as biomarkers in immunotoxicology. Toxicology 86, 213-232.

Wick, A., Jacobs, B., Kunkel, U., Heininger, P., Ternes, T.A., 2016. Benzotriazole UV stabilizers in sediments, suspended particulate matter and fish of German rivers: new insights into occurrence, time trends and persistency. Environ. Pollut. 212, 401-412.

Winnicki, A., Stańkowska-Radziun, M., Radziun, K., 1970. Structural and mechanical changes in the egg membranes of Salmo gairdneri Rich. during the period of hatching of the larvae Acta Ichthyologica Et Piscatoria 1, 7-20.

Zhang, J., Laurence Souders, C., Denslow, N.D., Martyniuk, C.J., 2017. Quercetin, a natural product supplement, impairs mitochondrial bioenergetics and locomotor behavior in larval zebrafish (Danio rerio). Toxicol. Appl. Pharmacol. 327, $30-38$.

Zhang, Z., Ren, N., Li, Y.F., Kunisue, T., Gao, D., Kannan, K., 2011. Determination of benzotriazole and benzophenone UV filters in sediment and sewage sludge. Environ. Sci. Technol. 45, 3909-3916.

Zhao, X., Zhang, Z.F., Xu, L., Liu, L.Y., Song, W.W., Zhu, F.J., Li, Y.F., Ma, W.L., 2017. Occurrence and fate of benzotriazoles UV filters in a typical residential wastewater treatment plant in Harbin, China. Environ. Pollut. 227, 215-222.

Zhuang, S., Lv, X., Pan, L., Lu, L., Ge, Z., Wang, J., Wang, J., Liu, J., Liu, W., Zhang, C., 2017. Benzotriazole UV 328 and UV-P showed distinct antiandrogenic activity upon human CYP3A4-mediated biotransformation. Environ. Pollut. 220, 616-624.

Zou, Y., Zhang, Y., Han, L., He, Q., Hou, H., Han, J., Wang, X., Li, C., Cen, J., Liu, K., 2017. Oxidative stress-mediated developmental toxicity induced by isoniazide in zebrafish embryos and larvae. J. Appl. Toxicol. 37, 842-852. 